\title{
Spin Bias Measurement Based on a Quantum Point Contact
}

\author{
Yanxia Xing and Qing-feng Sun* \\ Institute of Physics, Chinese Academy of Sciences, Beijing 100080, China. \\ Jian Wang \\ Department of Physics and the center of theoretical and computational physics, \\ The University of Hong Kong, Hong Kong, China.
}

\begin{abstract}
Electron charge transport through a quantum point contact (QPC) driven by an asymmetric spin bias (SB) is studied. A large charge current is induced when the transmission coefficient of the QPC jumps from one integer plateau to the next. Furthermore, for an open external circuit, the induced charge bias instead of the charge current is found to be quite large. It provides an efficient and practical way to detect SB by using a very simple device, a QPC or a STM tip. In addition, with the aid of magnetic field, polarization direction of the SB can also be determined.
\end{abstract}

PACS numbers: 85.75.-d, 85.30.Hi, 73.63.Rt, 73.23.-b

*Electronic address: sunqf@aphy.iphy.ac.cn 
The field of spintronics has received more and more attention recently. The spin current is one of the most important physical quantities in spintronics, 11, 2] similar to the role of the charge current played in electronics. Important issues of spintronics include how to generate, manipulate, and detect the spin current. Various methods have been proposed to generate spin current. [3, 4, 5, 6, 7, 8] Up to now, the spin current has been generated by various means, e.g., the pump excitation, [4] the optical injection, [5] the magnetic injection [6, 7], and the spin Hall effect. [8] To measure the spin current, the light-emitting diode or Kerrrotation spectroscopy has been experimentally used to detect the spin-current induced spin accumulation near the boundaries of the sample. [8] Moreover, an electric measurement was also realized via the inverse spin Hall effect.[7] In addition, theoretical proposals on indirect measurement of spin current were also available. [9, 10, 11, 12] These include the detection of the spin torque caused by a spin current flowing through a ferromagnetic-nonmagnetic interface[9] and the detection of the spin-current induced electric field.[10, 11] However, all these experimental instruments are delicate and complicated. They always involve the optical and magnetic factors, or spin-orbit interaction. Up to now, there is yet a practical and effective approach to measure spin current. Hence the measurement of the spin current remains a challenge.

In charge transport, one can measure the charge bias instead of the charge current. For a spin current flowing through a device, a spin-dependent chemical potentials (spin bias, $\mathrm{SB}$ ) is usually induced that is the driving force of the spin current. We can measure this SB instead of spin current. In this paper, we propose an effective method to detect the SB by using a quantum point contact (QPC) or a STM tip. The QPC is the simplest device in mesoscopic physics. The transport property of the QPC has been investigated extensively. Its conductance versus the gate voltage $V_{g}$ shows a series of step structures at the value $2 n e^{2} / \hbar(n=1,2, \ldots)$. Experimentally, the QPC has been used as a charge sensing detector to reliably probe the number of electrons in the quantum dot.[13] Our results show that a charge bias or charge current emerges when the QPC is biased by an asymmetric SB. [14] Therefore by measuring the induced charge bias, we can detect the SB in a very simple way.

The proposed device is a QPC device under an asymmetric SB as shown in Fig.1a.[15] Our task is to "experimentally" measure the SB. A concrete example of the device is shown in Fig.1c that is based on the experimental setup used in Ref. 7] where a ferromagnetic (FM) lead crossed over an aluminium (Al) lead. A charge current is injected from the FM lead 
into one terminal of $\mathrm{Al}$ lead, then a pure spin current is generated and flows into another terminal of the Al lead. A spin-dependent chemical potentials (i.e. SB) is created there. [7] Of course, the SB can also be generated using other methods. In this paper, we propose to measure this SB by using a STM tip (see Fig.1c) or a QPC device.

Let us first discuss the working principle of detecting the SB using the QPC. Due to the asymmetric SB $V_{s},[14]$ the chemical potentials on the left lead are spin-dependent with $\mu_{L \uparrow}=E_{f}+V_{s}$ and $\mu_{L \downarrow}=E_{f}-V_{s}$, but the chemical potentials on the right lead are still spin independent with $\mu_{R \uparrow}=\mu_{R \downarrow}=E_{f}$ (see Fig.1a). Under the SB $V_{s}$, the charge current $I_{\sigma}$ (with $\left.\sigma=\uparrow, \downarrow\right)$ is given by the Landauer-Büttiker formula:

$$
I_{\sigma}=\frac{e}{h} \int d E T(E)\left[f_{L \sigma}(E)-f_{R \sigma}(E)\right]
$$

where $T(E)$ is the transmission coefficient and $f_{p \sigma}=1 /\left\{\exp \left[\left(E-\mu_{p \sigma}\right) / k_{B} \mathcal{T}\right]+1\right\}$ is the Fermi distribution of the leads. From Eq.(1), the spin-up and spin-down currents, $I_{\sigma}$ with $\sigma=\uparrow, \downarrow$ or $\sigma= \pm$ are mainly determined by $T(E)$ with $E$ between $\mu_{R \sigma}=E_{f}$ and $\mu_{L \sigma}=E_{f}+\sigma V_{s}$. Note that the transmission coefficient $T(E)$ is energy dependent. In particular, when the energy $E$ is at the middle of the jump as shown in Fig.1b, $T(E)$ is strongly dependent on $E$. In general, $\left|I_{\uparrow}\right|$ is not equal to $\left|I_{\downarrow}\right|$. As a result, a net charge current $I_{c}=I_{\uparrow}+I_{\downarrow}$ occurs although $I_{\uparrow}$ and $I_{\downarrow}$ flow in opposite directions. So by measuring the induced charge current $I_{c}$, we can detect the $\mathrm{SB} V_{s}$. In the following, we shall investigate $I_{c}$ as well as the relation between $I_{c}$ and $V_{s}$ in detail.

To calculate $I_{c}$, we assume the QPC device is described by the Hamiltonian $H=\left(p_{x}^{2}+\right.$ $\left.p_{y}^{2}\right) / 2 m^{*}+V(x, y)$. In the QPC region $(|x| \leq L / 2$ and $|y| \leq W / 2)$, the potential is assumed in a saddle form $V(x, y)=\beta \frac{y^{2}}{W^{2}}-\frac{V_{g}}{\cosh ^{2}(\alpha x / L)^{2}}$. In the lead regions $(|x|>L / 2$ and $|y| \leq$ $\left.W_{\text {lead }} / 2\right), V(x, y)=0 . \quad V(x, y)=\infty$ outside of lead and QPC regions. In the tightbinding representation, the Hamiltonian becomes: 16] $H=-t \sum_{<\mathbf{i}, \mathbf{j}>, \sigma} a_{\mathbf{i} \sigma}^{\dagger} a_{\mathbf{j} \sigma}+\sum_{\mathbf{i}, \sigma}\left(4 t+V_{\mathbf{i}}\right) a_{\mathbf{i} \sigma}^{\dagger} a_{\mathbf{i} \sigma}$, where $\mathbf{i}=\left(i_{x}, i_{y}\right)$ labels the site index, $t=\hbar^{2} / 2 m^{*} a^{2}$ is the nearest neighbor hopping matrix element, $a$ is the distance between two neighboring sites, and $V_{\mathbf{i}}=V\left(i_{x} a, i_{y} a\right)$. From the discretized Hamiltonian $H$, the transmission coefficient $T(E)$ is given by $T(E)=$ $\operatorname{Tr}\left[\boldsymbol{\Gamma}_{L} \mathbf{G}^{r} \boldsymbol{\Gamma}_{R} \mathbf{G}^{a}\right]$ where we have used the Green function $\mathbf{G}^{r}(E)=\left[\mathbf{G}^{a}\right]^{\dagger}=\left\{E \mathbf{I}-\mathbf{H}_{0}-\right.$ $\left.\sum_{p} \boldsymbol{\Sigma}_{p}^{r}\right\}^{-1}$ and the line-width function $\boldsymbol{\Gamma}_{p}=i\left(\boldsymbol{\Sigma}_{p}^{r}-\boldsymbol{\Sigma}_{p}^{r \dagger}\right)$. Here $\mathbf{H}_{0}$ is the Hamiltonian of the QPC's region and $\Sigma_{p}^{r}$ is the retarded self-energy due to the coupling between the lead $p$ and the QPC. With $T(E)$, the charge current can be calculated from Eq.(1). 
In the numerical calculation, we set the hopping strength $t=1$ as the energy unit. The width of the leads is set to $W_{\text {lead }}=500 a$, and the QPC sizes are chosen as $W=50 a$ and $L=100 a$. The parameters $\alpha, \beta$ of the QPC's saddle potential are $\alpha=5$ and $\beta=4 t$. The Fermi energy $E_{f}$ is fixed at $0.6 t$ that is near the band bottom 0. Fig.2(a) and (b) show the charge current $I_{c}$ versus the gate voltage $V_{g}$ at different SB $V_{s}$ and at different temperatures $\mathcal{T}$, respectively. For comparison, the transmission coefficient $T(E)$ at $E=E_{f}$ versus $V_{g}$ is also plotted in Fig.2(b) (see gray solid line) which exhibits a series of step structures. For parameters we used, the curve of $T\left(E_{f}\right)-V_{g}$ is very similar to the curve of $T(E)$ - $E$ at fixed $V_{g}$ (not shown here). In the system, the charge current $I_{c}$ indeed is nonzero for nonzero SB $V_{s}$. From Fig.2 we see that $I_{c^{-}} V_{g}$ curves exhibit a series of peaks with approximately the same peak height. In addition these peaks are well correlated with $d T / d V_{g}$. For instance, $I_{c}$ reaches the maximum value whenever the transmission coefficient $T$ shows a jump. With increasing of $V_{s}$, the current $I_{c}$ increases as a whole (see Fig.2a). When increasing the temperature $\mathcal{T}$ from zero, $I_{c}$ near the peak decreases while $I_{c}$ near the valley increases. As a result, the curve of $I_{c}$ versus $V_{g}$ is smoothed out by temperature effect.

Experimentally, it is more convenient to measure the voltage. In the following, we consider an open external circuit where a charge bias $V_{c}$ is induced instead of the charge current $I_{c}$ passing through. Due to the induced charge bias $V_{c}$, the chemical potential $\mu_{R \sigma}$ of the right lead is shifted from $E_{f}$ to $E_{f}+V_{c}$. With condition $I_{c}=0$ in the open circuit, $V_{c}$ can be determined through Eq.(1). In Fig.3a and b, the ratio $V_{c} / V_{s}$ are plotted with the same parameters used in Fig2a and b. We see $V_{c} / V_{s}$ has similar characteristics as $I_{c}: V_{c} / V_{s}$ exhibits a series of peaks, increases with increasing $V_{s}$, and it is also smoothed out by the temperature effect. However, there are two big differences between $I_{c}$ and $V_{c}$. First, the peak heights of $V_{c} / V_{s}$ decrease as $\left|V_{g}\right|$ decreases, while they are nearly same for $I_{c}$. Second, on the left side of the first peak (at $V_{g} \approx-0.56$ ), $I_{c}$ is very small but $V_{c} / V_{s}$ is always quite large.

Fig.3c shows the ratio $V_{c} / V_{s}$ versus $V_{s}$ at different gate voltages $V_{g}$. When $V_{s}$ increases from the zero, $V_{c} / V_{s}$ rises quickly and then saturates eventually. We emphasize that the ratio $V_{c} / V_{s}$ is very sensitive to $V_{s}$ at small $V_{s}$. For instance $V_{c} / V_{s}$ is larger than 0.1 at $V_{s}=0.002 t$. Fig.3d gives the effect of temperature on the bias ratio $V_{c} / V_{s}$. We see that the peak of $V_{c} / V_{s}$ decreases while the valley of $V_{c} / V_{s}$ increases with the temperature. In the high temperature limit, $V_{c} / V_{s}$ converge to a constant value for all $V_{s}$. Notice that the high temperature limit 
of $V_{c} / V_{s}$ is quite large $(\approx 0.1)$. This means that the $\mathrm{SB} V_{s}$ can be measured even at high temperatures.

Let us discuss the feasibility of our proposal. First, the induced charge bias $V_{c}$ is quite large. $V_{c} / V_{s}$ can be over 0.5 at the certain region (e.g. while $V_{g}<-0.57$ as shown in Fig.3). In particular, $V_{c} / V_{s}$ is larger than 0.01 for almost all regions of the parameter including the SB $V_{s}$, the gate voltage $V_{g}$, the temperature $\mathcal{T}$, etc. In the present technology, the charge bias of order of $0.1 n V$ can easily be measured.[17] Therefore, our QPC device can detect $V_{s}$ if it is over $10 n V$. In fact, in the Ref.[7] the $\mathrm{SB} V_{s}$ has been estimated to be on the order of $10 \mu \mathrm{V}$ which is three order of magnitude larger than $10 \mathrm{nV}$.

So far we have discussed how to detect the magnitude of the SB. However, the SB is a vector with its magnitude and spin polarized direction.[18] Its polarized direction can be determined in the following way. We apply a magnetic field $\mathbf{B}$ to the QPC. Due to the Zeeman effect the transmission coefficient $T_{\sigma}(E)$ becomes spin-dependent. Note that $T_{\uparrow}(E)$ is increased and $T_{\downarrow}(E)$ is decreased when $\mathbf{B}$ is nonzero, where $\sigma=\uparrow, \downarrow$ represent the parallel and antiparallel direction of $\mathbf{B}$, respectively. As a result, the induced charge current $I_{c}$ or charge bias $V_{c}$ is also affected. By varying the direction of magnetic field $\mathbf{B}, I_{c}$ or $V_{c}$ reaches maximum value when the direction of $\mathbf{B}$ is parallel to the spin polarized direction of the SB $V_{s}$. Therefore the direction of $V_{s}$ can be determined.

In summary, when an asymmetric spin bias (SB) is applied on a quantum point contact device or a STM tip, a charge current or charge bias is induced. The SB can be determined by measuring the induced charge current or charge bias. Our analysis shows that the induced charge bias is quite large for almost all parameters and is well within the reach of the present technology. Hence our proposed device can efficiently measure the magnitude of the SB by an electrical measurement. In addition, the spin polarization direction of the SB can also be measured in the presence of magnetic field.

This work is supported by NSF-China under Grant Nos. 10525418, 10734110, and 60776060, and a RGC Grant (No. HKU 7048/06P) from the gov. of HKSAR. 
[1] S. A. Wolf, D. D. Awschalom, R. A. Buhrman, J. M. Daughton, S. von Molnr, M. L. Roukes, A. Y. Chtchelkanova, and D. M. Treger, Science 294, 1488 (2001); G. A. Prinz, Science 282, 1660 (1998).

[2] I. Žutic, J. Fabian, S. Das Sarma, Rev. Mod. Phys. 76, 323 (2004).

[3] Q.-F. Sun, H. Guo, and J. Wang, Phys. Rev. Lett. 90, 258301 (2003); W. Long, Q.-F. Sun, H. Guo and J. Wang, Appl. Phys. Lett. 83, 1397 (2003); B. Wang, J. Wang, J. Wang, and D. Y. Xing, Phys. Rev. B 69, 174403 (2004); A. W. Cummings, R. Akis, and D. K. Ferry, Appl. Phys. Lett. 89, 172115 (2006); F. Chi and J. Zheng, Appl. Phys. Lett. 92, 062106 (2008).

[4] S. K. Watson, R. M. Potok, C. M. Marcus and V. Umansky, Phys. Rev. Lett. 91, 258301 (2003).

[5] J. Hübner, W. W. Rühle, M. Klude, D. Hommel, R. D. R. Bhat, J. E. Sipe, and H. M. van Driel, Phys. Rev. Lett. 90, 216601 (2003); M. J. Stevens, A. L. Smirl, R. D. R. Bhat, A. Najmaie, J. E. Sipe and H. M. van Driel, Phys. Rev. Lett. 90, 136603 (2003); X. D. Cui, S.-Q. Shen, J. Li, Y. Ji, W. Ge, F.-C. Zhang, Appl. Phys. Lett. 90, 242115 (2007).

[6] T. Kimura, Y. Otani, T. Sato, S. Takahashi and S. Maekawa, Phys. Rev. Lett. 98, 156601 (2007); E. Saitoh, M. Ueda, H. Miyajima and G. Tatara, Appl. Phys. Lett. 88, 182509 (2006).

[7] S. O. Valenzuela and M. Tinkham, Nature 442, 176 (2006).

[8] Y. K. Kato, R. C. Myers, A. C. Gossard and D. D. Awschalom, Science 306, 1910 (2004); J. Wunderlich, B. Kaestner, J. Sinova and T. Jungwirth, Phys. Rev. Lett. 94, 047204 (2005).

[9] P. Mohanty, G. Zolfagharkhani, S. Kettemann and P. Fulde, Phys. Rev. B 70, 195301 (2004); T.-W. Chen, C.-M. Huang and G. Y. Guo, Phys. Rev. B 73, 235309 (2006).

[10] Q.-F. Sun, H. Guo and J. Wang, Phys. Rev. B 69, 054409(2004); Q.-F. Sun and X. C. Xie, Phys. Rev. B 72, 245305 (2005).

[11] F. Meier and D. Loss, Phys. Rev. Lett. 90, 167204 (2003); F. Schütz, M. Kollar and P. Kopietz, Phys. Rev. Lett. 91, 017205 (2003).

[12] Q.-F. Sun, Y. Xing, and S.-Q. Shen, Phys. Rev. B 77, 195313 (2008); H.-Z. Lu and S.-Q. Shen, Phys. Rev. B 77, 235309 (2008).

[13] W. G. van der Wiel, S. De Franceschi, J. M. Elzerman, T. Fujisawa, S. Tarucha and L. P. Kouwenhoven, Rev. Mod. Phys. 75, 1 (2003). 
[14] D.-K. Wang, Q.-F. Sun and H. Guo, Phys. Rev. B 69, 205312 (2004).

[15] The asymmetric SB is crucial in our proposal since we need the non-zero charge current. If a symmetric SB with $\mu_{L \uparrow}=-\mu_{L \downarrow}=-\mu_{R \uparrow}=\mu_{R \downarrow}=V_{s}$ is applied to the QPC, the induced charge current $I_{c}$ is always zero and our method does not apply. In fact, in most cases, the $\mathrm{SB}$ is asymmetric. For example, in the proposed experimental setup in Fig.1c, the chemical potential in the STM is always spin-independent, so there is always an asymmetric SB.

[16] Y. Xing, Q.-F. Sun, L. Tang and J. P. Hu, Phys. Rev. B 74, 155313 (2006);

[17] H. Safar, P. L. Gammel, D. A. Huse, D. J. Bishop, J. P. Rice and D. M. Ginsberg, Phys. Rev. Lett. 69, 824 (1992).

[18] Q.-F. Sun, X. C. Xie, and J. Wang, Phys. Rev. B 77, 035327 (2008). 
FIG. 1: (Color online) (a) Schematic plot of an asymmetric SB applying on a QPC. (b) The transmission coefficient $T(E)$ vs the energy $E$ for a typical QPC device. In (a) and (b), the spindependent chemical potentials $\mu_{L \sigma}$ are also shown. (c) Schematics of a suggested device is shown where a FM lead crossed over an Al lead. A spin current is injected into the Al lead and a SB is generated along the $\mathrm{Al}$ lead. A tip of STM as a detector to detect the SB.

FIG. 2: (Color online) (a) $I_{c}$ vs $V_{g}$ at zero temperature $\mathcal{T}$ for $V_{s}=0.005$ (solid line), 0.01 (dashed line), and 0.02 (dotted line). (b) $I_{c}$ vs $V_{g}$ at fixed $V_{s}=0.02$ for $k_{B} \mathcal{T}=0$ (solid line), 0.005 (dashed line), and 0.01 (dotted line). For comparison, $T\left(E_{f}\right)$ vs $V_{g}$ is also plotted in (b), [see gray (or red) solid line].

FIG. 3: (Color online) (a) and (b) are $V_{c} / V_{s}$ vs $V_{g}$ for different SB $V_{s}$ (a) and different temperature $k_{B} T$ (b). (c) is $V_{c} / V_{s}$ vs $V_{s}$ for different $V_{g}$, and (d) is $V_{c} / V_{s}$ vs $\mathcal{T}$ for different $V_{g}$. The other parameters are same to that in Fig.2. 
(a)

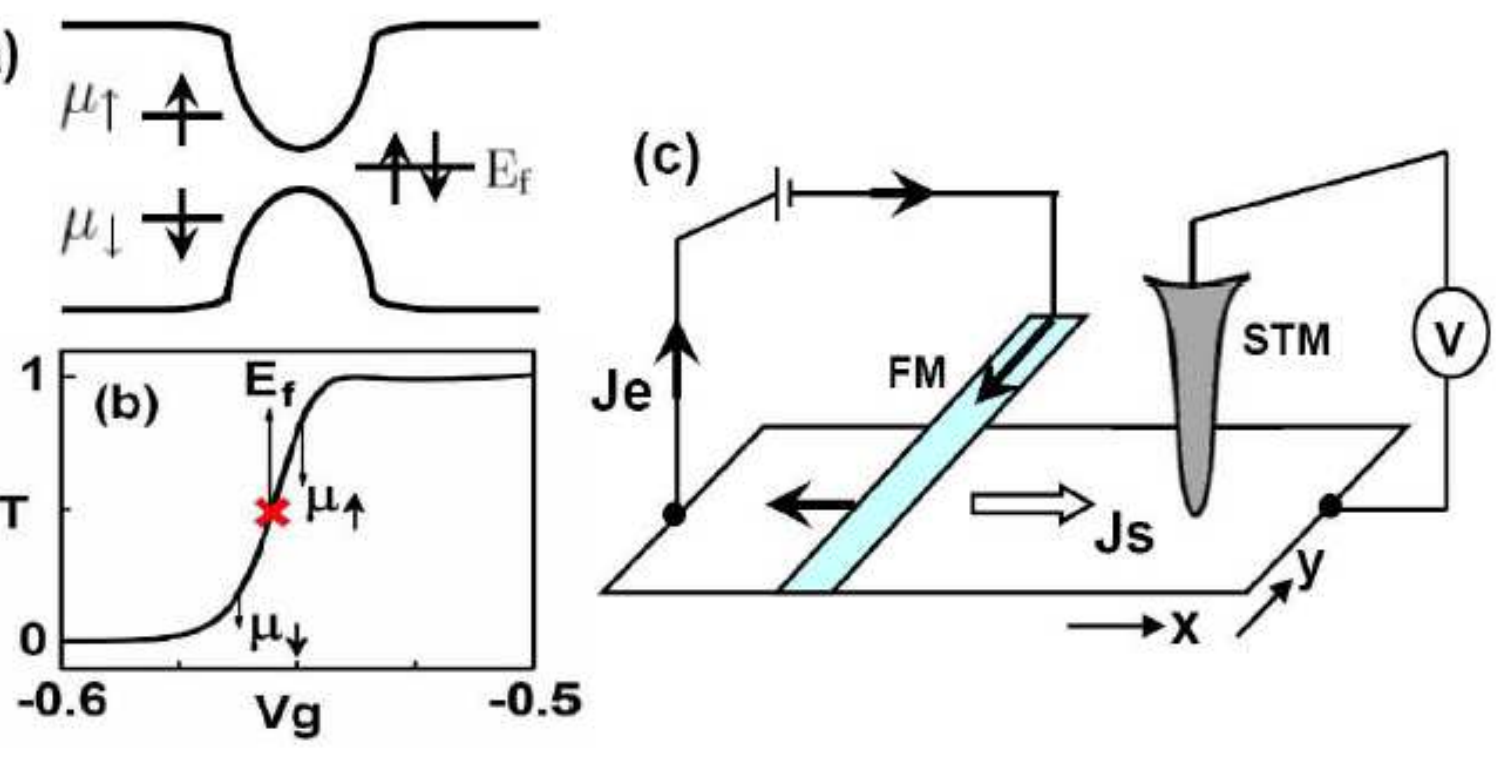




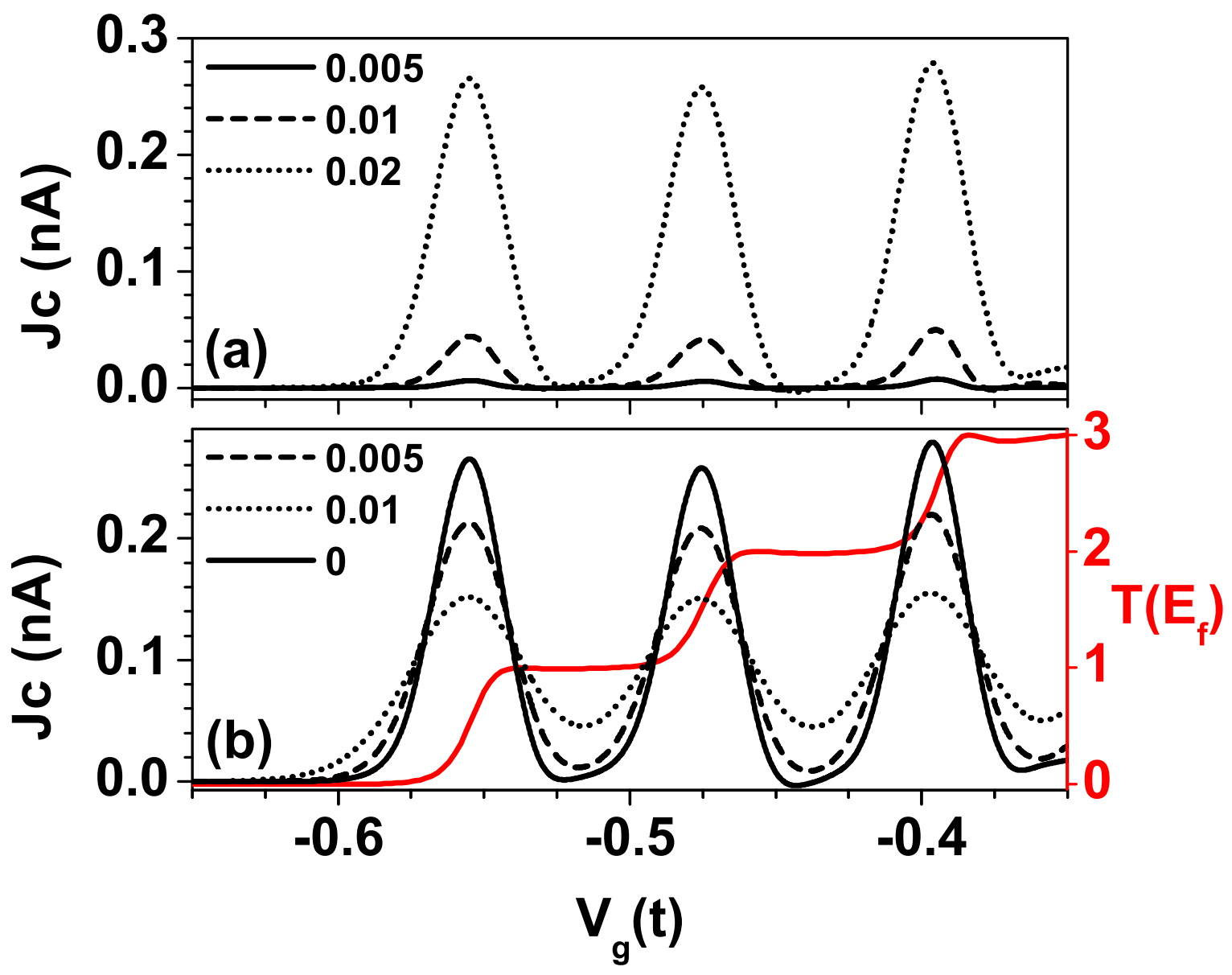



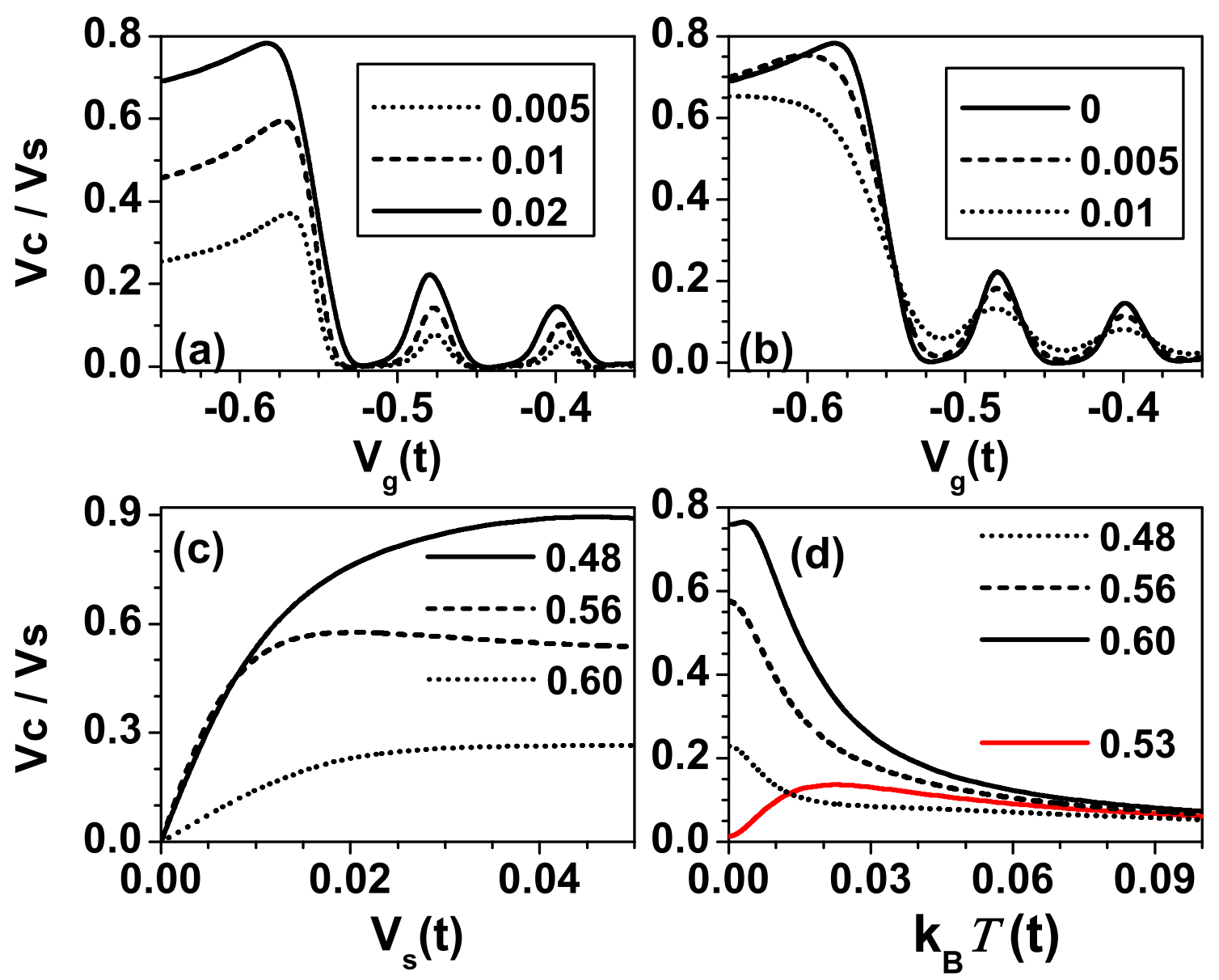